\title{
Phase-controlled slow and fast light in current-modulated semiconductor optical amplifiers
}

\author{
M.A. Antón, F. Carreño, Óscar G. Calderón, Sonia Melle, \\ Francisco Arrieta-Yáñez \\ Escuela Universitaria de Óptica, Universidad Complutense de Madrid, C/ Arcos \\ de Jalón s/n, 28037 Madrid, Spain \\ E-mail: antonm@fis.ucm.es
}

\begin{abstract}
We present a theoretical study of the slow and fast light propagation in semiconductor optical amplifiers based on coherent population oscillations. By modulating the injection current to force the population oscillations we can modify the delay or advancement of light signals. Specifically, it is shown that the relative phase of the optical signal to the bias current modulations can be used as a knob for changing light propagation from delay to advancement. In addition, we analyze the effect of the modulation current for slow light in vertical cavity surface emission lasers (VCSELs) by taking into account the cavity effects. It is shown that the change of the depth of the modulation allows to tune the structural resonance, which in turn produces an enhancement of the delay.
\end{abstract}

PACS numbers: 42.65.-k,42.50.Nn,42.70.Nq,42.55.Px 


\section{Introduction}

Recent dramatic experimental demonstration of slow and fast light has stimulated considerable interest in the dynamic control of the group velocity of light and in the development of tunable all-optical delays for applications such as optical buffers. Two methods are generally exploited to control optical delay: one of them relies in the use of dispersive devices and the other is based on the modification of the group index of an optical medium. The first approach is structural, where one aims for finding an optimal structure that enhances the nonlinear response (through its geometrical properties). Some of the most promising systems that explore this approach are FabryPerot resonators, high Q cavities, and photonic crystals. The second approach makes use of nonlinear optical effects such as electromagnetically induced transparency (EIT) 1. 2, 3, 4, coherent population oscillation (CPO) [5], Raman [6, 7] and Brillouin amplification [8, 9, 10]. These nonlinear optical techniques have been extensively studied in atomic systems, however the corresponding counterparts in solid state crystals, fibers, and semiconductors are extremely attractive in order to obtain optical tunable and easily integrable devices like delay lines, and buffers. EIT in a solid has been demonstrated with a rare-earth doped matrix [11] and in a Pr-doped solid at 5 $\mathrm{K}$ [12. Some of the coherent effects which are present in dilute systems have been also investigated theoretically and experimentally in semiconductors [13, 14, 15]. In particular, EIT was experimentally obtained using exciton and biexciton transitions in a quantum-well (QW) structure [16, 17, 18, 19].

Coherent population oscillations have been shown to be a robust physical mechanism which allows for the variation of group velocity. CPO produces a narrow hole in the absorption or gain profile as a consequence of the periodic modulation of the ground state population at the beat frequency between a strong control field and a weak probe field sharing a common atomic transition. Unlike EIT, which is dominated by the coherence dephasing time, $\mathrm{CPO}$ is governed by the population relaxation time and becomes nearly insensitive to temperature. In addition, CPO is weakly influenced by inhomogeneous broadening in atomic systems in contrast to the quantum coherence effect involved in EIT. Slow and fast light at room temperature originated by CPO has been experimentally observed in solid state crystals [5, 20, 21], erbium doped fibers (EDFs) [22, 23, 24], photorefractive materials [25, 26], and biological thin films [27, among others.

Slow and fast light in semiconductor optical amplifiers (SOAs) has also been studied extensively in recent years 28, 29, because those such systems have the advantage of providing compactness, easy integration with electronic or optical systems, large bandwidth due to fast carrier dynamics, and easy and quick tuning of delay by direct current injection or optical pumping. When a strong control beam and a weak signal beam (at different wavelengths) propagate through a SOA, beating between the two beams causes oscillations of the carrier density. These oscillations create dynamical gain and index gratings in the device. Interaction of the signal beam with the dynamical gratings results in the group index change experienced by the signal. The group index can be controlled either electrically (by changing the bias current of the SOA) or optically (by changing the pump power). Using this method, a group index reduction of 10 has been demonstrated in a compact $2 \mathrm{~mm}$ device. CPO based slow light has also been reported in a multiple-quantum-well structure at low temperature [13] and in quantum-dot (QD) semiconductor optical amplifiers operating at $1.3 \mu \mathrm{m}$ at room temperature [30]. Recently, Su et al reported 
that the four-wave mixing (FWM) effect, in conjunction with the CPO effect, plays an important role in a quantum-well SOA in the gain regime 31. Furthermore, slow light at room temperature and with a bandwidth in the range of $\mathrm{GHz}$ can be achieved in semiconductor quantum wells and quantum dots 32. All these results can be understood by considering the theory of CPO and FWM (see 33, 34 and references therein for a review). It has been shown that the delay or advancement achieved saturates with both pump and signal powers and is limited by the carrier lifetime. However, the effects of the refractive index mediated by wave mixing can be exploited to increase the degree of light control by optical filtering prior to detection [35. In the conventional CPO studies in SOAs, the optical beam is modulated in the RF range and delay or advancement of the detected signals are measured depending on the value of the DC bias current, according to be below or above the transparency current. Therefore, current modulation is another mechanism which can be externally controlled to modify the semiconductor optical response. Here, we will analyze the possibility of realization of slow and fast light in a SOA by considering the simultaneous modulation of the optical beam and the bias current in such a medium. The dynamics of SOAs subject to direct current modulation has not been dealt in the slow light context. The feasibility to periodically modulate the bias current in the RF range has been previously addressed [36] from a theoretical point of view. The possibility to modify at will the relative phase of the modulated bias current to the probe field allows us to manage the magnitude of the delay or advancement experienced by the probe field.

\section{Theoretical model of forced population oscillations}
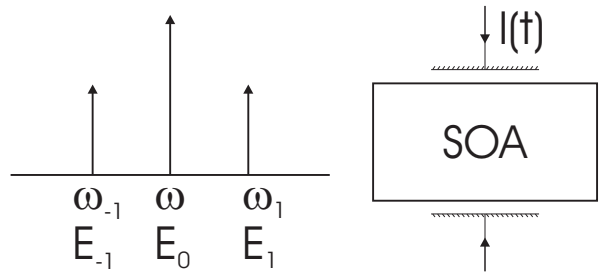

Figure 1. Current modulated SOA and spectral components of the optical field impinging on it. The angular frequencies of the sidebands are detuned from $\omega$ by $\delta: \omega_{ \pm 1}=\omega \pm \delta$

We consider a SOA driven by an injected current $I$. A laser field couples the transition between the semiconductor valence band and the conduction band. Typical spontaneous carrier lifetimes are in the order of a few nanoseconds. By adjusting the SOA injection current we may achieve an amplifying/absorbing semiconductor medium at relatively low injection currents, $I$, ranging from a few $\mathrm{mA}$ to several hundred of $\mathrm{mA}$.

To model the population oscillation in the semiconductor structure, the theoretical starting point relies on considering that an optical beam $\mathcal{E}(t)$ impinges 
on the medium. The optical field is given by

$$
\mathcal{E}(t)=\frac{1}{2} E(t) e^{-i \omega t}+\text { c.c. },
$$

$\omega$ being the angular frequency of the optical field and $E(t)$ is the slowly-varying amplitude. We assume that this field is comprised of a strong DC control beam $E_{0}$ and two sidebands $E_{1}$ and $E_{-1}$ separated by the modulation angular frequency $\delta$ which lies within the RF range. The explicit expression for $E(t)$ is given by

$$
E(t)=E_{0}(t)+E_{1}(t) e^{-i \delta t}+E_{-1}(t) e^{i \delta t} .
$$

When this modulated beam goes through the SOA (see figure1), the three components of the electrical field interact with the carriers in the semiconductor through stimulated emission and will impose a modulation on the carrier density due to the frequency beating between the optical waves. When the beating frequency $\delta$ is small enough that the carriers in the SOA can follow the oscillation between the valence band and the conduction band, the carriers will generate a temporal grating and induce the energy exchange between the control and the sideband fields. This process creates a spectral hole seen by the sideband fields with width on the order of $\mathrm{GHz}$ (inverse of the carrier lifetime).

The carrier density $N$ attained for a selected bias current $I$, is obtained by solving the following rate equation:

$$
\frac{d N}{d t}=\frac{I}{q V}-\frac{N}{\tau}-\frac{1}{2} n_{b g} c \epsilon_{0} \frac{\Gamma g(N)}{\hbar \omega_{0}}\left\langle|E(t)|^{2}\right\rangle,
$$

where $q$ is the electron charge, $V$ is the active volume, $g(N)$ is the modal gain experienced by the optical field when the current $I$ is injected into the active region of the semiconductor amplifier, and $\Gamma$ is the confinement factor, that is, the fraction of mode energy confined within the active volume $V, \omega_{0}$ is the angular frequency between the semiconductor valence band and the conduction band, $\tau$ is the carrier lifetime, and $n_{b g}$ is the background refractive index of the material. The angle brackets denote the averaging operation over the active volume. Equation (3) can be derived from the density-matrix equations [37, 38, in the rate-equation approximation. In equation (3) the effects of carrier diffusion in the transverse direction have been ignored since the transverse dimensions of the SOA are generally smaller than the diffusion length. In addition, a linear modal gain $g(N)$ is assumed to be equal for all the involved waves, an assumption justified since the pump-probe detuning is much smaller compared to the gain-spectrum bandwidth, i.e., $\delta \tau \ll 1$, then the following condition is satisfied:

$$
g(N)=\alpha\left(N-N_{t}\right)
$$

$\alpha$ being the gain cross-section and $N_{t}$ is the carrier density at which the active region becomes transparent. These equations neglect ultrafast gain nonlinearities like carrier heating and spectral hole burning, which is a good approximation for moderate modulation frequencies below $20 \mathrm{GHz}[36$.

In the conventional CPO studies in SOAs, the optical beam is modulated in the $\mathrm{RF}$ range and delay or advancement of the detected signals are measured depending on the value of the DC injection current, according to be below or above the transparency current. Here, we will analyze the possibility of improving slow and fast light performance in SOAs by considering the simultaneous modulation of the optical beam 
and the bias current in such a medium. Both magnitudes are modulated at the same frequency $\delta$. The feasibility to produce modulations in the bias current in the RF range has been previously addressed from a theoretical point of view [36].

Our goal is to force the population oscillations by modulating the bias current $I(t)$, which is described as:

$$
I(t)=I_{0}+I_{+1} e^{-i(\delta t-\Psi)}+I_{-1} e^{i(\delta t-\Psi)},
$$

where $I_{ \pm 1} \ll I_{0}$, and it is assumed that the modulated current could be out of phase with respect to the modulation of the optical weak probe field by a magnitude $\Psi$, which can be externally changed. In what follows we also assume that the current is independent on the spatial coordinates, i.e., we will consider a traveling microwave which will be matched exactly with the propagating optical fields. When the modulated beam propagates through the SOA, the three components of the electrical fields interact with one another and result in a relative phase shift. The interaction of the sidebands and the control beam causes CPO, modifies the temporal refractive index, and changes the group velocity of the light signal. Simultaneously, the injected current and optical beams are also coupled to each other through the wave-mixing effects, resulting in an additional phase shift.

To solve equation (3), we substitute $E(t), g(N)$ and $I(t)$ from equations (2), (4) and (5), respectively, which results in the following equation:

$$
\begin{aligned}
\frac{d N}{d t}= & -\frac{N}{\tau}+\frac{N_{t}}{\tau}\left(R_{0}+R_{1} e^{-i(\delta t-\Psi)}+R_{-1} e^{i(\delta t-\Psi)}\right) \\
& -\frac{\left(N-N_{t}\right)}{\tau P_{\text {sat }}} \frac{1}{2} n_{b g} c \epsilon_{0}\left[\left|E_{0}\right|^{2}+\left(E_{0}^{*} E_{1}+E_{0} E_{-1}^{*}\right) e^{-i \delta t}+\left(E_{0} E_{1}^{*}+E_{0}^{*} E_{-1}\right) e^{i \delta t}\right]
\end{aligned}
$$

where we have defined the following normalized currents $R_{0, \pm 1}$ and the saturation power $P_{\text {sat }}$ :

$$
\begin{aligned}
& R_{0, \pm 1}=\frac{\tau}{q V N_{t}} I_{0, \pm 1}, \\
& P_{\text {sat }}=\frac{\hbar \omega_{0}}{\Gamma \alpha \tau} .
\end{aligned}
$$

Next we assume that the carrier density can be described by a DC term and small $\mathrm{AC}$ terms modulated at the same beating frequency, i.e.,

$$
N(t)=N_{0}+N_{1} e^{-i \delta t}+N_{-1} e^{i \delta t},
$$

where $N_{0}$ is the static carrier density and $N_{ \pm 1}$ is the amplitude of the carrier population oscillation of the corresponding sideband. The solution of equation (6) yields the following expressions for the carrier density amplitudes:

$$
\begin{aligned}
& N_{0}=N_{t} \frac{R_{0}+q_{0}}{1+q_{0}} \\
& N_{1}=N_{t} \frac{R_{1} e^{i \Psi}-\left(N_{0} / N_{t}-1\right) q_{1}}{1+q_{0}-i \delta \tau}, \\
& N_{-1}=\left(N_{1}\right)^{*}
\end{aligned}
$$

where we have defined the following normalized optical powers: 


$$
\begin{aligned}
q_{0} & =\frac{1}{2} n_{b g} c \epsilon_{0} \frac{\left|E_{0}\right|^{2}}{P_{\text {sat }}}, \\
q_{1} & =\frac{1}{2} n_{b g} c \epsilon_{0} \frac{\left(E_{0}^{*} E_{1}+E_{0} E_{-1}^{*}\right)}{P_{\text {sat }}} .
\end{aligned}
$$

In view of the previous considerations, we arrive at the following equation for the carrier density oscillation:

$$
N(t)=N_{0}+N_{t}\left[\frac{R_{1} e^{i \Psi}-\left(N_{0} / N_{t}-1\right) q_{1}}{1+q_{0}-i \delta \tau} e^{-i \delta t}+\text { c.c. }\right] .
$$

A close inspection of equation (15) reveals that population oscillation works as a temporal grating whose amplitude depends both on the coupling between the DC and the sidebands of the probe field, and on the modulation term of the injection current $R_{1}$. The oscillating part of equation (15) contains two terms: one, which is proportional to $q_{1}$, is responsible for the conventional population oscillation observed up to date 38 and arises from the modulation of the field connecting the optical transition. The other one, which is proportional to $R_{1}$, arises from the modulation imposed to the bias current and incorporates explicitly the relative phase $\Psi$. We will show that this term is responsible for the probe delay/advancement enhancement.

The response of the system to the weak probe field can be obtained by solving the scalar wave equation:

$$
\nabla^{2} \mathcal{E}(z, t)-\frac{1}{c^{2}} \frac{\partial^{2} \mathcal{E}(z, t)}{\partial t^{2}}=\frac{1}{c^{2} \epsilon_{0}} \frac{\partial^{2} \mathcal{P}(z, t)}{\partial t^{2}} .
$$

The field given by equation (11) induces a complex polarization in the medium

$$
\mathcal{P}(z, t)=\frac{1}{2}\left(P_{0}(z)+P_{1}(z) e^{-i \delta t}+P_{-1}(z) e^{i \delta t}\right) e^{-i(\omega t-k z)}+\text { c.c. },
$$

where $P_{i}(z)(i=0,1,-1)$ is a complex polarization coefficient that yields index and absorption characteristics for the DC and side modes waves. It is well-known [38, that the induced polarization is given by

$$
\mathcal{P}(z, t)=-\frac{c \epsilon_{0}(\beta+i)}{\omega(1-i \Delta)} \alpha\left(N(t)-N_{t}\right) \mathcal{E}(z, t),
$$

where $\beta$ stands for the so-called linewidth enhancement factor and $\Delta=\omega_{0}-\omega$ is the detuning. Introducing equation (15) and (11) into equation (18) allows us to obtain the components of the polarization $P_{i}(z)$, which are given by

$$
\begin{aligned}
P_{0}(z)= & -\frac{c \epsilon_{0}(\beta+i) \alpha N_{t}}{\omega(1-i \Delta) \omega_{c}}\left[\left(R_{0}-1\right) E_{0}\right. \\
& \left.+\frac{\left(\omega_{c} R_{1} e^{i \Psi}-\left(R_{0}-1\right) q_{1}\right)}{\omega_{c}-i \delta \tau} E_{-1}+\frac{\left(\omega_{c} R_{1} e^{-i \Psi}-\left(R_{0}-1\right) q_{1}^{*}\right)}{\omega_{c}+i \delta \tau} E_{1}\right], \\
P_{1}(z)= & -\frac{c \epsilon_{0}(\beta+i) \alpha N_{t}}{\omega(1-i \Delta) \omega_{c}}\left[\left(R_{0}-1\right) E_{1}+\frac{\left(\omega_{c} R_{1} e^{i \Psi}-\left(R_{0}-1\right) q_{1}\right)}{\omega_{c}-i \delta \tau} E_{0}\right], \\
P_{-1}(z)= & -\frac{c \epsilon_{0}(\beta+i) \alpha N_{t}}{\omega(1-i \Delta) \omega_{c}}\left[\left(R_{0}-1\right) E_{-1}+\frac{\left(\omega_{c} R_{-1} e^{-i \Psi}-\left(R_{0}-1\right) q_{1}^{*}\right)}{\omega_{c}+i \delta \tau} E_{0}\right] .
\end{aligned}
$$


We have defined the dimensionless frequency $\omega_{c}=1+q_{0}$ which roughly measures the linewidth of the transparency hole created in the absortion/gain spectrum due to CPO. Now we substitute equations (19) in equation (16), and by equating the coefficients oscillating at the same frequency, we arrive at the following set of equations for the amplitudes of the optical fields in the SVEA approximation:

$$
\begin{aligned}
\frac{\partial E_{0}}{\partial z}= & \frac{\alpha N_{t}(1-i \beta)}{2 \omega_{c}}\left[\left(R_{0}-1\right) E_{0}+\frac{\left(\omega_{c} R_{1} e^{i \Psi}-\left(R_{0}-1\right) q_{1}\right)}{\omega_{c}-i \delta \tau} E_{-1}\right. \\
& \left.+\frac{\left(\omega_{c} R_{1} e^{-i \Psi}-\left(R_{0}-1\right) q_{1}^{*}\right)}{\omega_{c}+i \delta \tau} E_{1}\right], \\
\frac{\partial E_{1}}{\partial z}= & \frac{\alpha N_{t}(1-i \beta)}{2 \omega_{c}}\left[\left(R_{0}-1\right) E_{1}-\frac{\left(R_{0}-1\right) q_{1}}{\omega_{c}-i \delta \tau} E_{0}+\frac{\omega_{c} R_{1} e^{i \Psi}}{\omega_{c}-i \delta \tau} E_{0}\right], \\
\frac{\partial E_{-1}}{\partial z}= & \frac{\alpha N_{t}(1-i \beta)}{2 \omega_{c}}\left[\left(R_{0}-1\right) E_{-1}-\frac{\left(R_{0}-1\right) q_{1}^{*}}{\omega_{c}+i \delta \tau} E_{0}+\frac{\omega_{c} R_{1} e^{-i \Psi}}{\omega_{c}+i \delta \tau} E_{0}\right],
\end{aligned}
$$

where we have assumed the probe field to be at resonance for simplicity, i.e., $\Delta=0$. It is well known that the first two terms appearing in the equation of evolution of the sidebands lead to coherent dips in pump-probe spectroscopy 38. The first one is related to the linear susceptibility of each sideband, while the second term arises from the multiple wave mixing process. This second term is responsible for the creation of a hole in the probe gain and therefore is the physical origin of conventional CPO. Here, we should notice that the last term that contributes to the development of the sidebands arises from a net exchange from the DC component of the optical field which in turn arises as a consequence of the modulation of the current $\left(R_{1}\right)$. In the case that $\Psi=0$, the contribution of this term will produce an in phase contribution to the index and gain gratings that will result in an enhancement/fall of the CPO depending on the value of the bias current to be below or above the transparency level. In the case that $\Psi=\pi$, it will produce a change that will turn delay into advancement. In the general case that $\Psi \neq 0, \pi$, the last term will result in a mixing of the gain grating and the index grating which is responsible for the enhancement of the phase delay/advancement. Note that the equation for the DC component also incorporates the effects of the weak sidebands as source terms.

These effects can become more transparent by considering the spatial propagation of the magnitude $q_{1}$ which accounts for detection of the output modulated signal. By neglecting the spatial variation in $E_{0}$ (non-depleted approximation) and $\beta=0$ we arrive to the following expressions for its modulus and phase:

$$
\begin{aligned}
\frac{d\left|q_{1}\right|}{d z}= & -\frac{\alpha N_{t} q_{0}}{2 \omega_{c}}\left[\left(R_{0}-1\right) \frac{\left|q_{1}\right|}{q_{0}}\left(1-\frac{2 q_{0} \omega_{c}}{\omega_{c}^{2}+(\delta \tau)^{2}}\right)\right. \\
& \left.+\frac{2 \omega_{c} R_{1}}{\omega_{c}^{2}+(\delta \tau)^{2}}\left(\omega_{c} \cos \Psi-\delta \tau \sin \Psi\right)\right], \\
\frac{d \phi}{d z}= & -\frac{\alpha N_{t} q_{0}}{\omega_{c}\left[\omega_{c}^{2}+(\delta \tau)^{2}\right]}\left[\left(R_{0}-1\right) \delta \tau-\frac{\omega_{c} R_{1}}{\left|q_{1}\right|}\left(\omega_{c} \sin \Psi+\delta \tau \cos \Psi\right)\right],
\end{aligned}
$$

A close inspection of equation (21) reveals that the four-wave mixing effect cancels out in this detection scheme. In this case, and in the absence of current modulation 
$\left(R_{1}=0\right)$, the slow-down effect reduces to a saturation phenomenon. However, the non null value of current modulation will produce a resonance behavior which mimics the effects of a four-wave mixing process.

It is worth mentioning that equation (21) reduces to those obtained by Mørk et al 36, with the proper identifications of variables, except for the fact that in our case we consider input optical fields at the entrance of the medium (at $z=0$ ).

\section{Effect of current modulation on the phase delay/advancement}
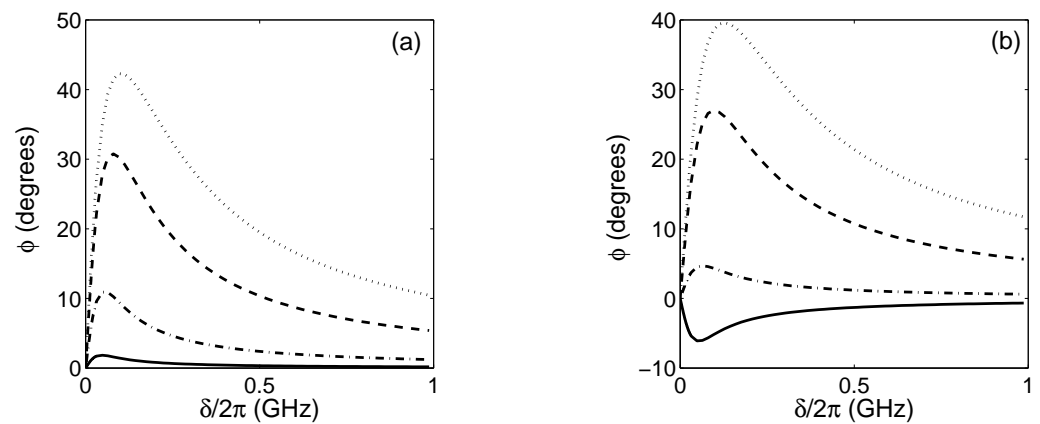

Figure 2. Phase delay versus the modulation frequency for different values of the modulation depth: $R_{1}=0$ (solid line), $R_{1}=0.01 \times R_{0}$ (dashed-dotted line), $R_{1}=0.05 \times R_{0}$ (dashed line), and $R_{1}=0.1 \times R_{0}$ (dotted line). DC injection current (a) $R_{0}=0.95$ (b) $R_{0}=1.15$. Other parameters are: $q_{0}=0.5$, $q_{1}=0.1 \times q_{0}$, and $\Psi=0$.

In this section we will perform numerical simulations concerning the influence of current modulation on slow and fast light in a SOA. For this purpose we will carry out numerical integration of equation (20) for selected values of the parameters involved, which may be accessible from an experimental point of view. To illustrate the effect of the current modulation we use the following parameters: the overlap factor $\Gamma$ is assumed to be 1 , the linewidth enhancement factor $\beta=0$, the effective carrier lifetime $\tau=5 \mathrm{~ns}$, the transparent carrier density $N_{t}=1 \times 10^{18} \mathrm{~cm}^{-3}$, and the length $L=0.3$ $\mathrm{mm}$. We also assume that the gain coefficient is $\alpha=2 \times 10^{-16} \mathrm{~cm}^{2}$, and use $n_{b g}=3.2$ as the background refractive index. By considering a typical area in the order of $10^{-12}$ $\mathrm{m}^{2}$, the saturation power is $P_{\text {sat }}=4.27 \mathrm{~mW}$. The theoretical operation of the SOA should have a threshold current (transparency current) in the order of $I_{t}=6.4 \mathrm{~mA}$.

We present in figure 2(a) the results obtained for the case in which the injection current is below the transparency current $\left(R_{0}<1\right)$. In this case the overall effect of the modulation current is to produce a huge increase of the maximum phase delay for moderate values of the modulation while producing a slight increase of the bandwidth in the range of $1 \mathrm{GHz}$. The most remarkable effect is obtained when operating above the threshold injection current $\left(R_{0}>1\right)$ as it is shown in figure 2(b). There, we appreciate that for a moderate value of the modulation current (dashed-dotted curve) delay is achieved. The delay tends to increase over all the range of frequencies with regard to that obtained for the DC injection current case (solid line). A further increase of the modulation current turns gain into absorption which reflects in the obtention of phase delay over all the range of frequencies (dashed and dotted lines). Thus, the 
level of modulation of the current allows the control of the level of advancement and to switch from fast to slow light. In order to qualitatively explain this behavior we can use the trivial solution of equation (21) for the phase delay by neglecting the $z$-dependence of the rest of magnitudes:

$\phi(z=L) \simeq-\frac{\alpha L N_{t} q_{0}}{\omega_{c}\left[\omega_{c}^{2}+(\delta \tau)^{2}\right]}\left[\left(R_{0}-1\right) \delta \tau-\frac{\omega_{c} R_{1}}{\left|q_{1}\right|}\left(\omega_{c} \sin \Psi+\delta \tau \cos \Psi\right)\right]$.

In the case that $\Psi=0$ (in phase case), this phase delay (22) is proportional to $R_{0}-1-\omega_{c} R_{1} /\left|q_{1}\right|$, therefore, the current modulation contributes to produce delay. Thus, for an injection current below the transparency one, where delay is expected, the current modulation increases its magnitude. However, at an injection current above the transparency one, where advancement is expected, the current modulation produces a decrease of the advancement, and for a threshold value of the modulation amplitude $R_{1}=\left(R_{0}-1\right)\left|q_{1}\right| / \omega_{c}$, the phase advancement turns into delay. Therefore, the modulation amplitude $R_{1}$ can be used as a control parameter to switch the propagation regime.

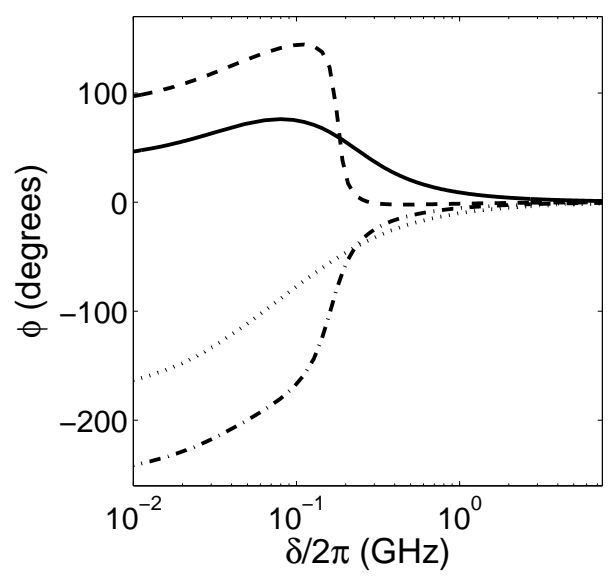

Figure 3. Phase delay versus the modulation frequency for different values of the relative phase $\Psi: \Psi=45^{\circ}$ (solid line), $\Psi=100^{\circ}$ (dashed line), $\Psi=120^{\circ}$ (dashed-dotted line), $\Psi=180^{\circ}$ (dotted line). Operating point $R_{0}=0.95$, subject to a modulation $R_{1}=0.1 \times R_{0}$. Other parameters as in figure 2

In order to show how the relative phase shift of the modulation current to the modulated optical field $(\Psi)$ influences the phase shift $\phi$, we plot in figure 3 the phase delay versus the beat frequency for several values of $\Psi$. We have selected an operating point below the transparency current $\left(R_{0}<1\right)$. Note that under these circumstances, by solely modifying the $\mathrm{AC}$ current $\left(R_{1}\right)$ and by keeping $\Psi=0$, we remain in the absorptive regime which always produces delay on the optical signal [see figure 2(a)]. However, when the modulation current is fixed while the relative phase is properly changed, a switch from absorption to gain is produced which results in turning delay into advancement of the optical signal. In other words, the phase $\Psi$ may be used as an external parameter to control the magnitude of phase delay/advancement achieved for all the range of frequencies while keeping constant the rest of parameters. The change in the regime of propagation has its counterpart in the change from absorption to gain 
(not shown). This behavior can be easily explained in terms of the new contributions to the index and gain gratings originated by the modulation current [see equation (21)]. For the values $\Psi=0$, and $\Psi=\pi$, the effect of current modulation only affects the absorptive grating, while for values like $\Psi=\pi / 4$ (dashed-dotted line), both the gain and the index gratings should contribute to the phase delay experienced by the optical signal. From equation (22), we can estimate a threshold value of $\Psi$ to switch from delay to advancement, i.e., $\Psi \simeq-\arctan \left(\delta \tau / \omega_{c}\right)+\arcsin \left[\left(R_{0}-1\right)\left|q_{1}\right| \delta \tau /\left(\omega_{c} R_{1}\right)\right]$.

Figure 4 shows examples of the calculated phase delay versus the relative phase $\Psi$ for two different values of the amplifier length $(L)$. In the case of small length (solid line), the phase delay exhibits a nearly sinusoidal behavior. This fact is consistent with the linear approximation which leads to equation (22). An increase of the length of the medium results in the breaking of the sinusoidal shape (see dashed-dotted line). This effect arises as a consequence of propagation effects associated to the numerical solution of equation (20).

In the previous calculations we have used a null value for the linewidth enhancement factor $(\beta)$. The influence of such parameter in the index change and the probe gain has been a subject of analysis in the pioneering work of Agrawal 38 . In that work, it was shown that the increment of $\beta$ will result in a distortion of the line shape. In the present case, where we are interested in the phase of the modulated signal intensity, the influence of $\beta$ on $\phi$ is unnoticeable, since the coherent population effect due to the pump beam dominates, while the four wave mixing effects cancel out.

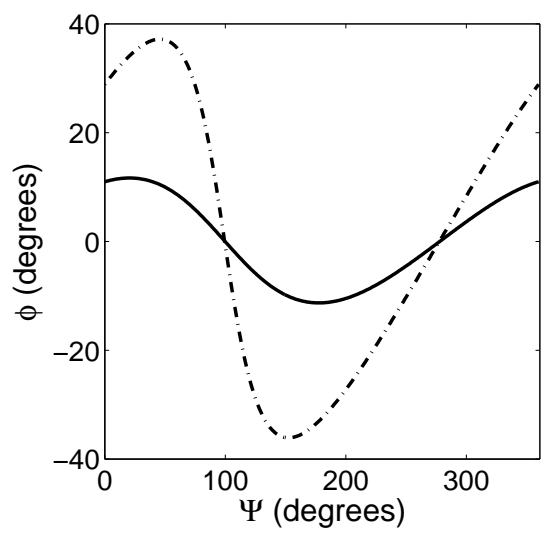

Figure 4. Phase delay versus the relative phase $\Psi$ for two values of the length of the active region: $L=0.1 \mathrm{~mm}$ (solid line) and $L=0.3 \mathrm{~mm}$ (dashed-dotted line), subject to a modulation $R_{1}=0.1 \times R_{0}$ for a modulation frequency $\delta /(2 \pi)=0.3$ GHz. Other parameters as in figure 2

\subsection{Slow and fast light in the sidebands by optical filtering}

In the conventional detection schema commonly used in many slow light experiments, no optical filtering is performed in the output beam of the SOA. However, Xue et al 35] have shown that the degree of control of the optical delay in a SOA can be improved by the application of an optical filtering of the output signal prior to detection. In the present case, we will also analyze how the current modulation will affect the delay or advancement when optical filtering is performed. Figure 5(a) presents the results 
obtained for a null linewidth enhancement factor $(\beta=0)$. There we appreciate that the blue-filtered signal (dashed line, filtering $E_{1}$ ) nearly coincides with the signal obtained without optical filtering (solid line), while the red-filtered signal (dashed-dotted line, filtering $E_{-1}$ ) exhibit advancement in contrast to the other two cases. The three curves exhibit a symmetric line shape. This behavior can be explained by simply considering that the four wave mixing terms does not cancel out. The situation is dramatically modified when considering a non-null value of $\beta$ as it is displayed in figure5(b). In this case the blue-filtered, the red-filtered and the non filtered values for $\phi$ obtained differ among them, due to the combined effect of the linewidth enhancement factor and the modulation current term which is proportional to $R_{1}$ [see equation (20)]. Numerical simulations carried out (not shown) reveal that the greater the value of $\beta$, the greater the magnitude of the phase delay/advancement obtained. These results agree with the ones obtained in [38] where the magnitude of the asymmetry in the index and gain change for the probe was shown to be $\beta$-dependent.
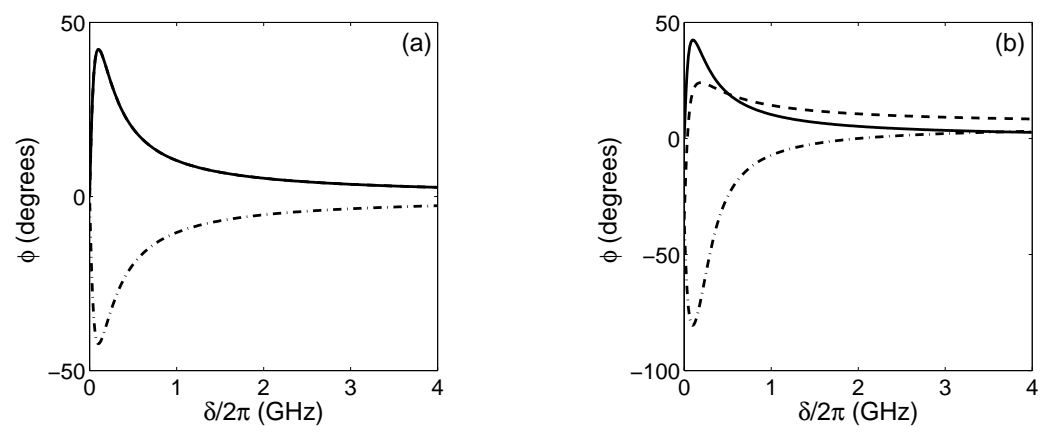

Figure 5. Phase delay versus the modulation frequency with $\beta=0$ (a) and with $\beta=1$ (b). Solid line (without optical filtering $q_{1}$ ), dashed-dotted line (by filtering $E_{-1}$ ), and dashed line (by filtering $E_{1}$ ), at a fixed value of the modulation depth $R_{1}=0.1$ and DC injection current $R_{0}=0.95$, and $\Psi=0$. Other parameters as in figure 2

Now we fix the detuning to $\delta / 2 \pi=0.5 \mathrm{GHz}$ and will allow for a change of the relative phase $\Psi$. The results are displayed in figure 6 for the three possible cases of the output signals. It is worth noting that the peak values of delay/advancement achieved for the filtered signals are greater than the ones obtained for the non-filtered case. This result resembles that obtained in [35] in the sense that in the mentioned paper the authors showed that the filtered signals were shown to exhibit greater delays than the non-filtered signal, although in that case the authors changed the input power while we keep the input power fixed in our simulations.

Figure 7 presents the results obtained versus the depth of the modulation $\left(R_{1}\right)$ at the same fixed detuning as before but now the phase of the modulating current to the modulated field is also fixed at $\Psi=\pi$. We appreciate that the unfiltered signal shows a switch from advancement to delay whereas the filtered signals for both sidebands exhibit the largest delay for the highest values of the modulating current, leading to a saturation effect for values larger than 0.4. For other values of the relative phase $\Psi$ the behavior is similar to that displayed in figure (7) although the level of phase delay/advancement remains within the same limiting values. 


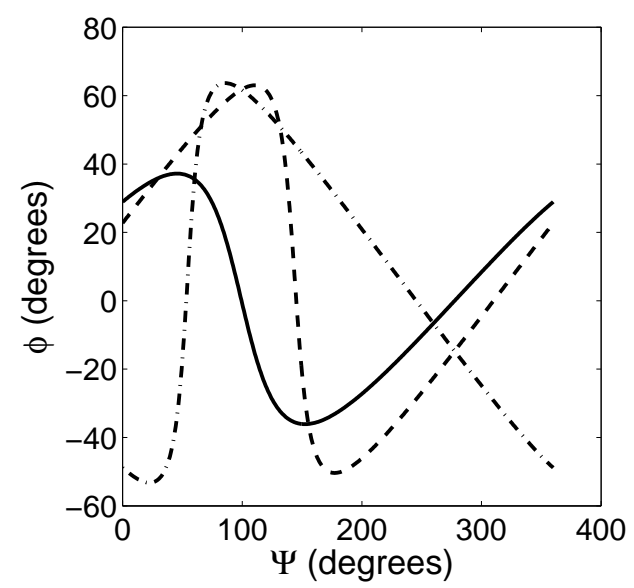

Figure 6. Phase shift $\phi$ versus relative phase $\Psi$. Solid line (without optical filtering $q_{1}$ ), dashed-dotted line (by filtering $E_{-1}$ ), and dashed line (by filtering $E_{1}$ ), at a fixed value of the modulation depth $R_{1}=0.1 \times R_{0}, \delta / 2 \pi=0.3 \mathrm{GHz}$, DC injection current $R_{0}=0.95$, and $\beta=1$.

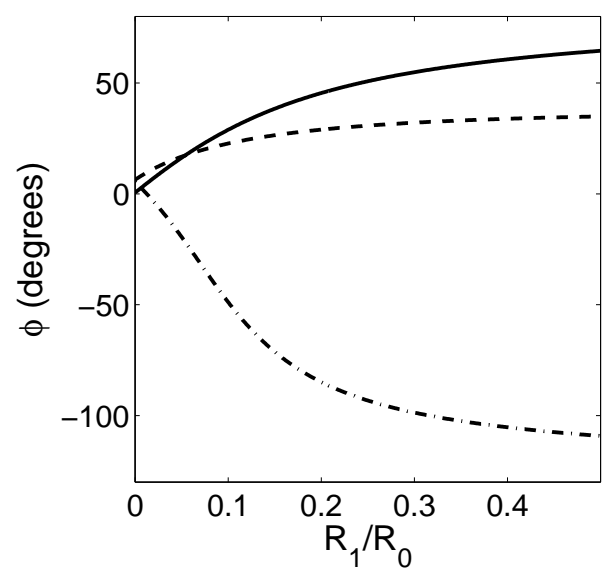

Figure 7. Phase shift $\phi_{s}$ versus $R_{1} / R_{0}$. Solid line (without optical filtering $q_{1}$ ), dashed-dotted line (by filtering $E_{-1}$ ), and dashed line (by filtering $E_{1}$ ) at a fixed of $\Psi=0$. Other parameters as in figure 6

\subsection{Cavity enhanced slow light in a VCSEL}

Tunable delay using VCSELs has been demonstrated [41. In addition, the temporal dynamics of VCSELs subject to direct current modulations have also received attention. Verschaffelt et al [42] have addressed how the current modulation forces the population oscillations. Here we turn our attention to extend our previous model to the case of such a device. The high reflectivities of the mirrors in the VCSEL will require to include the cavity effects in the optical response of the medium together with the population oscillations. A simple way to incorporate both effects relies in the consideration of the VCSEL as a Fabry-Perot filter with a gain per pass. This approach 
can be justified by considering the small longitudinal dimensions of the device which are in the order of a few micrometers. For the sake of simplicity, we will also consider that the effects of the intrinsic birefringence of the medium can been neglected, thus we can use the scalar wave equations given in equation (20). This approach has been adopted in several studies concerning slow light in these devices 43, 44. In view of the previous considerations, the reflectance $G_{r}$ obtained for the filter, is given by [43]

$$
G_{r}=\frac{\left(\sqrt{R_{t}}+\sqrt{R_{b}} g_{s}\right)^{2}+4 \sqrt{R_{t}} \sqrt{R_{b}} g_{s} \sin ^{2}[p h i]}{\left(1-\sqrt{R_{t} R_{b}} g_{s}\right)^{2}+4 \sqrt{R_{t}} \sqrt{R_{b}} g_{s} \sin ^{2}[\phi]},
$$

where $R_{b} / R_{t}$ stands for the bottom/top mirror reflectance, $g_{s}$ is the single-pass gain, and $\phi$ is the single-pass phase delay obtained. The magnitudes $g_{s}$ and $\phi$ are obtained from the numerical solution obtained from equation (20). Figure 8 presents the numerical results obtained for the phase of $G_{r}, \psi_{r}$, by considering an active region of length $L=1.13 \mu \mathrm{m}$. The reflectances of the top and bottom mirrors are assumed to be $R_{t}=0.997$, and $R_{b}=0.99$, respectively. Note that for this particular modulation frequency, the changes in the depth of the bias current modulation results in changes from one regime of propagation to the other. The most remarkable feature seen in figure 8 is the appearance of a resonance whose origin is attributed to the tuning of a mode in the Fabry-Perot filter. The change of the depth of the modulation allows to tune the resonance, which in turn produces an enhancement of the delay.

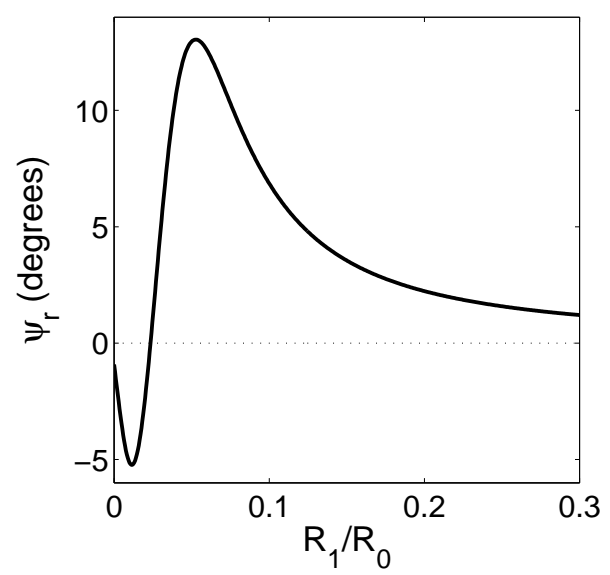

Figure 8. Phase delay $\psi_{r}$ at the output of the VCSEL versus modulation depth $R_{1} / R_{0}$ at a fixed modulation frequency of $\delta / 2 \pi=0.05 \mathrm{GHz}$, DC injection current $R_{0}=0.95, \Psi=0$, and $\beta=0$.

\section{Conclusions}

In this work we have presented numerical simulations concerning to the enhancement of the delay/advancement based in CPO in SOA when the bias current is modulated at the same beating frequency. An overall increase of delay/advancement is obtained for all the frequency range. The depth of the modulation, and the reference phase are shown to have a dramatic influence on the magnitude of the phase delay. The slow and fast light have been also considered for the case of filtering the output optical 
fields prior to the detection. Significant changes in the phase delay are predicted by adjusting the relative phase or the modulation depth for the filtered signals. The model is also extended to a VCSEL by adding the cavity effects arising from the hight reflectivities Bragg mirrors of the device. In this case, it is also shown that the modulation depth can be used to tune the structural resonance of the device modeled as a Fabry-Perot filter, which results in a phase delay of the output signal.

\section{Acknowledgments}

This work has been supported by Projects no. PR34/07-15847 (UCM/BSCH), FIS2007-65382 (MEC), CCG07-UCM/ESP-2179 (UCM-CM), CCG08-UCM/ESP4220 from Spain.

\section{References}

[1] Boyd R W and Gauthier D J 2002 Slow and fast light (Progress in Optics vol 43) ed E Wolf (Amsterdam: Elsevier) chap 6 p 497-530

[2] Hau L V, Harris S E, Dutton Z and Behroozi C H 1999 Nature 397594

[3] Kash M M, Sautenkov V A, Zibrov A S, Hollberg L, Welch G R, Lukin M D, Rostovtsev Y, Fry E S and Scully M O 1999 Phys. Rev. Lett. 825229

[4] Wang L J, Kuzmich A and Dogariu A 2000 Nature 406277

[5] Bigelow M S, Lepeshkin N N and Boyd R W 2003 Science 301200

[6] Dahan D and Eisenstein G 2005 Opt. Express 136234

[7] Sharping J, Okawachi Y, van Howe J, Xu C, Willner A, Wang Y and Gaeta A 2005 Opt. Express 137872

[8] Okawachi Y, Bigelow M S, Sharping J E, Zhu Z, Schweinsberg A, Gauthier D J, Boyd R W and Gaeta A L 2005 Phys. Rev. Lett. 94153902

[9] Sharping J, Okawachi Y and Gaeta A 2005 Opt. Express 136092

[10] Song K Y, Herraez M G and Thevenaz L 2005 Opt. Express 1382

[11] Ham B S and Hemmer P R 2000 Phys. Rev. Lett. 844080

[12] Turukhin A V, Sudarshanam V S, Shahriar M S, Musser J A, Ham B S and Hemmer P R 2002 Phys. Rev. Lett. 88023602

[13] Ku P-C, Sedgwick F, Chang-Hasnain C J, Palinginis P, Li T, Wang H, Chang S W and Chuang S L 2004 Opt. Lett. 292291

[14] Palinginis P, Sedgwick F, Crankshaw S, Moewe M. and Chang-Hasnain C. 2005 Opt. Express 139909

[15] Su H and Chuang S L 2006 Opt. Lett. 31271

[16] Phillips M and Wang H 2002 Phys. Rev. Lett. 89186401

[17] Phillips M and Wang H 2003 Opt. Lett. 28831

[18] Serapiglia G B, Paspalakis E, Sirtori C, Vodopyanov K L, Phillips C C 2003 Phys. Rev. Lett. 841019

[19] Silvestri L, Bassani F, Czajkowski G and Davoudi B 2002 Eur. Phys. J. B. 2789

[20] Bigelow M S, Lepeshkin N N and Boyd R W 2003 Phys. Rev. Lett. 90113903

[21] Baldit E, Bencheikh K, Monnier P, Levenson J A and Rouget V 2005 Phys. Rev. Lett. 95143601

[22] Zhang Y, Qiu W, Ye J, Wang N, Wang J, Tian H and Yuan P 2008 Opt. Commun. 281 2633; Zhang Y, Qiu W, Ye J, Wang N, Wang J, Tian H, Wang H and Yuan P 2008 Phys. Lett. A 3722724

[23] Schweinsberg A, Lepeshkin N N, Bigelow M S, Boyd R W and Jarabo S 2006 Europhys. Lett. 73218

[24] Melle S, Calderón O G, Carreño F, Cabrera E, Antón M A and Jarabo S 2007 Opt. Commun. 279 53; Melle S, Calderón O G, Caro C E, Cabrera-Granado E, Antón M A and Carreño F 2008 Opt. Lett. 33 827; Calderón O G, Melle S, Antón M A, Carreño F and Arrieta-Yáñez F 2008 Phys. Rev. A $\mathbf{7 8} 053812$

[25] A. Shumelyuk, K. Shcherbin, S. Odulov, B. Sturman, E. Podivilov, K. Buse Phys. Rev. Lett. 93 (2004) 243604.

[26] Zhang G, Bo F, Dong R and Xu J 2004 Phys. Rev. Lett. 93133903

[27] Wu P and Rao D V (2005) Phys. Rev. Lett. 95253601

[28] Mørk J, Kjaer R, van der Poel M, and Yvind K 2005 Opt. Express 138136 
[29] Öhman F, Yvind K, and Mørk J 2006 Opt. Express 149955

[30] Su H and Chuang S L 2006 Appl. Phys. Lett. 88061102

[31] Su H, Kondratko P and Chuang S L 2006 Opt. Express 2006144800

[32] Chang S W, Kondratko P K, Su H and Chuang S L 2007 IEEE J. Quantum electron. 43196

[33] Ku P C, Chang-Hasnain C J and Chuang S L 2007 J.Phys. D: Appl. Phys. 40 R93

[34] Pesala B, Sedgwick F, Uskov A and Chang-Hasnain C J 2008 J. Opt. Soc. Am. B 25 C46

[35] Xue W, Chen Y, Öhman F, Sales S and Mørk J 2008 Opt. Lett. 331084

[36] Mørk J, Mecozzi A and Eisenstein G 1999 IEEE J. Sel. Top. Q. Electr. 5851

[37] Sargent III M, Zhou F and Koch S W 1988 Phys. Rev. A 384673

[38] Agrawal G P 1988 J. Opt. Soc. Am. B 5147

[39] Boyd R W, Raymer M G, Narum P and Harter D J 1981 Phys. Rev. A 24411

[40] Boyd R W, Gauthier D J and Gaeta A L 2006 Opt. Photon. News 1718

[41] Zhao X, Palinginis P, Pesala B, Chang-Hasnain C J, Hemmer P 2005 Opt. Express 137899

[42] Verschaffelt G, Alvert J, Veretennicoff I, Danckaert J, Barbay S, Giacomelli G, Marin F 2002 Appl. Phys. Lett. 802248

[43] Laurand N, Calvez S and Dawson M D 2006 Opt. Express 146858

[44] Chou C-S, Lee R-K, Peng P-C, Kuo H-C, Lin G, Yang H P and Chi J Y 2008 J. Opt. A: Pure Appl. Opt. 10044016 